\title{
Foam Structure Preservation during Microwave-Assisted Vacuum Drying: Significance of Interfacial and Dielectric Properties of the Bulk Phase of Foams from Polysorbate 80-Maltodextrin Dispersions
}

\author{
Peter Kubbutat $^{1, *(\mathbb{D})}$, Ulrich Kulozik ${ }^{1}$ (D) and Jannika Dombrowski ${ }^{1,2}$ \\ 1 Chair of Food and Bioprocess Engineering, TUM School of Life Science, Technical University of Munich, \\ Weihenstephaner Berg 1, 85354 Freising, Germany; Ulrich.Kulozik@tum.de (U.K.); \\ Jannika.Dombrowski@rd.nestle.com (J.D.) \\ 2 Nestlé Research, Société des Produits Nestlé SA, Route du Jorat 57, 1000 Lausanne, Switzerland \\ * Correspondence: peter.kubbutat@tum.de
}

Citation: Kubbutat, P.; Kulozik, U.; Dombrowski, J. Foam Structure Preservation during

Microwave-Assisted Vacuum Drying: Significance of Interfacial and Dielectric Properties of the Bulk Phase of Foams from Polysorbate 80-Maltodextrin Dispersions. Foods 2021, 10, 1163. https://doi.org/ 10.3390 / foods 10061163

Academic Editors: Matt

Duncan Golding and Joana S. Amaral

Received: 24 March 2021

Accepted: 20 May 2021

Published: 22 May 2021

Publisher's Note: MDPI stays neutra with regard to jurisdictional claims in published maps and institutional affiliations.

Copyright: (C) 2021 by the authors Licensee MDPI, Basel, Switzerland. This article is an open access article distributed under the terms and conditions of the Creative Commons Attribution (CC BY) license (https:// creativecommons.org/licenses/by/ $4.0 /)$.

\begin{abstract}
This study aimed at examining the cause of differences in the structure preservation of polysorbate 80-maltodextrin foams during microwave-assisted vacuum drying (MWVD) versus conventional vacuum drying (CVD). Aqueous dispersions of $3 \%$ polysorbate 80 and $0-40 \%$ maltodextrin were characterized for their dielectric and interfacial properties, and results were related to their drying performance in a foamed state. Surface tension and surface dilatational properties as well as dielectric properties clearly responded to the variation in the maltodextrin content. Likewise, the foam structure preservation during CVD was linked to the maltodextrin concentration. Regarding MWVD, however, foams collapsed at all conditions tested. Nevertheless, if the structure during MWVD remained stable, the drying time was significantly reduced. Eventually, this finding could be linked to the dielectric properties of polysorbate 80 rather than its adsorption kinetics and surface film viscoelasticity as its resonant frequency fell within the working frequency of the microwave drying plant.
\end{abstract}

Keywords: vacuum drying; surfactant; polysaccharide; foam decay; resonant frequency

\section{Introduction}

For the preservation of heat-sensitive biological material (e.g., bacterial cultures [1,2] enzymes [3] or pharmaceutical ingredients [4]), vacuum drying has evolved into a promising alternative to time- and energy-intensive freeze drying. Besides advantages in the specific energy demand [5] and drying time [6], vacuum drying was shown to yield products of increased storage stability as compared to freeze drying. The increased storage stability was linked to product shrinkage during dehydration [7], which, however, entails a prolongation of the third drying stage. In addition, due to the compactness of the dried product structure, its grindability is impaired. In this context, Ambros et al. [8] reported that microwave assistance can reduce the vacuum drying time by about $95 \%$. This significant effect was related to the volumetric energy input of the microwaves allowing for a high mass transfer over all drying stages. Furthermore, controlled product aeration (i.e., foaming) prior to microwave-assisted vacuum drying (MWVD) was proposed to tackle the drawback of conventional vacuum drying (CVD) in terms of poor product grindability [9]. Additionally, the drying process can be accelerated by means of foaming. This can be attributed to the higher specific surface of an aerated versus a non-aerated product as well as the presence of lamellae, which act as capillaries during the drying process, transporting the water to the product's surface [10].

Overall, MWVD of foamed materials was shown to display a promising concept. However, it is important to note that product formulation (e.g., foaming agent, protec- 
tant, carrier) and characteristics (e.g., foam characteristics) as well as process conditions (e.g., pressure level, microwave power level, drying protocol) need to be well adapted and controlled [11]. Therewith, foam structure stability and thus product quality, besides process efficiency, are ensured. In order to avoid detrimental foam collapse during drying and further optimize MWVD in terms of the drying time and resulting product quality, a more in-depth understanding of the interdependency of the above-mentioned influencing factors is required [12]. It was assumed that interfacial and/or dielectric properties of the material to be dried would play a key role. Thereby, the former allow for conclusions regarding adsorption kinetics and the stability of the interfacial film against mechanical stress (e.g., stretching) and are associated with foamability and foam stability [13,14]. The latter provide insights into the interaction of the material to be dried with the electromagnetic field of the microwave and are key for process understanding [15]. To the authors' best knowledge, neither interfacial characteristics nor dielectric properties of surfactant-polysaccharide or protein-polysaccharide dispersions have been investigated in the context of microwave-assisted vacuum drying, thus far. Therefore, the objective of the present study was to evaluate these characteristics (i.e., surface tension and surface dilatational rheology, dielectric constant and loss factor, resonant frequency and quality factor) for polysorbate 80-maltodextrin dispersions and to correlate them with the samples' drying performance in terms of foam structure preservation during MWVD versus CVD. In this model system, the non-ionic surfactant polysorbate 80 served as a foaming agent, whereas the polysaccharide maltodextrin was used to enhance foam stability by means of increasing the bulk viscosity. In addition, maltodextrin is widely applied as a protectant or carrier for sensitive biological material during drying $[8,16,17]$. The behavior of whey protein-maltodextrin-based matrices was examined in a further study [18].

\section{Materials and Methods}

\subsection{Materials and Preparation of Sample Dispersions}

Sample dispersions of $200 \mathrm{~g}$ each were prepared by blending 3.0\% $w / w$ polysorbate 80 (Tween 80; Gerbu Biotechnik GmbH, Heidelberg, Germany) with different amounts (i.e., $0.0-40.0 \% w / w)$ of maltodextrin DE-6 (MD; Nutricia GmbH, Erlangen, Germany) and dissolving the dry mix in deionized water (Milli-Q Integral 3, Merck KGaG, Darmstadt, Germany). To ensure full hydration, the sample dispersions were gently stirred using a magnet stirrer (Maxi Direct, Fisher Scientific GmbH, Schwerte, Germany) at $200 \mathrm{rpm}$ for $12 \mathrm{~h}$ at $4{ }^{\circ} \mathrm{C}$. Prior to the experiments, the sample dispersions were tempered at $20^{\circ} \mathrm{C}$ with a water bath (F3, Fisher Scientific GmbH, Schwerte, Germany).

Unless mentioned otherwise, measurements were performed at $20^{\circ} \mathrm{C}$ and at least in triplicate using independent batches of sample dispersions. Error bars represent the calculated standard deviation.

\subsection{Surface Tension and Surface Dilatational Properties of the Bulk Phase}

Time-dependent evolution of surface tension $\sigma$ was determined by the pendant drop method using the DSA100R (Krüss GmbH, Hamburg, Germany). A drop of $12 \mu \mathrm{L}$ was formed at the tip of a capillary $\left(\mathrm{d}_{\mathrm{i}}=1.81 \mathrm{~mm}\right.$, Krüss $\mathrm{GmbH}$, Hamburg, Germany) and its contour was monitored for $120 \mathrm{~min}$. Extraction of surface tension was conducted by drop shape analysis using the software Advance (Krüss GmbH, Hamburg, Germany).

Upon reaching quasi-equilibrium surface tension, surface dilatational properties were determined by the oscillating drop method. For this, the pendant drop at a drop age of $60 \mathrm{~min}$ was exposed to a sinusoidal oscillation with a frequency of $0.1 \mathrm{~Hz}$ for a duration of $100 \mathrm{~s}$. The amplitude was set to $200-800 \%$ in order to achieve a change in surface area by $2.5-3.5 \%$. Data fitting according to Lucasson and Van den Tempel [19] allowed for the estimation of surface dilatational elasticity $E^{\prime}$ and surface dilatational viscosity $E^{\prime \prime}$ :

$$
E=\frac{\Delta \sigma}{\Delta A / A_{0}}
$$




$$
\begin{aligned}
& E^{\prime}=|E| \cos \varphi \\
& E^{\prime \prime}=|E| \sin \varphi
\end{aligned}
$$

where $E$ is the complex viscoelastic modulus, $\Delta A / A_{0}$ is the amplitude of the surface area oscillation and $\varphi$ is the phase angle between surface tension oscillation and surface area oscillation. The tangent $\varphi$ was calculated by dividing $E^{\prime \prime} / E^{\prime}$ according to Conde et al. [20].

\subsection{Dielectric Properties of the Bulk Phase}

Dielectric properties of the sample dispersions were measured with the $\mu$ WaveAnalyser (Püschner GmbH \& Co. KG, Schwanewede, Germany) over a broad temperature range from -40 to $+40{ }^{\circ} \mathrm{C}$. For this, $300 \mu \mathrm{L}$ of sample dispersion was filled into a $1 \mathrm{~mL}$ glass vial, which was then sealed with a lock (1MLFBG, VWR International GmbH, Darmstadt, Germany). For each measurement point, the filled vial and the $\mu$ WaveAnalyser were tempered at the respective target temperature within a climate chamber. After an equilibration time of $6 \mathrm{~h}$, measurements were performed at a frequency between 2400 and $2500 \mathrm{MHz}$ against an empty vial. Based on the response of the sample dispersion towards the emitted frequency, the software $\mu$ WaveAnalyser Version 3.2.0 (Püschner GmbH \& Co. KG, Schwanewede, Germany) calculates the dielectric constant $\varepsilon^{\prime}$, the dielectric loss $\varepsilon^{\prime \prime}$, the resonant frequency $f_{\text {res }}$ and the quality factor $Q$ [21]. The dielectric constant is a measure of the polarizability of a material, whereas the dielectric loss is a parameter for the physical conversion of electromagnetic radiation into heat. In addition, the loss factor $\tan \delta$ describes the ability of the sample dispersion to take up energy from the electromagnetic field and is defined by the ratio of loss factor and dielectric constant [22]:

$$
\tan \delta=\frac{\varepsilon^{\prime \prime}}{\varepsilon^{\prime}}
$$

In addition, the penetration depth PD can be calculated according to Equation (5) [23]:

$$
P D=\frac{\lambda_{2450 M H z}}{2 \cdot \pi} \frac{\sqrt{\varepsilon^{\prime}}}{\mathcal{E}^{\prime \prime}}
$$

The penetration depth describes the depth until the power density has decreased to $\frac{1}{e}$ of its initial value and depends on the wavelength $\lambda$. In this study, the wavelength was assumed with $2450 \mathrm{MHz}$.

\subsection{Foam Preparation}

Foams were prepared by means of whipping with a commercial planetary mixer (KitchenAid ARTISAN 5KSM105PS, Whirlpool Corp., Greenville, United States of America) equipped with a wire whisk geometry (K45WW, Whirlpool Corp., Greenville, United States of America). Per foam, $150 \mathrm{~g}$ of sample dispersion was whipped for $15 \mathrm{~min}$ at $220 \mathrm{rpm}$. Immediately after foam formation, $15 \mathrm{~g}$ of foamed sample was gently transferred to a cylindrical crystallization glass with a diameter of $200 \mathrm{~nm}$ (VWR International GmbH, Darmstadt, Germany) and dried as described in Section 2.5.

The resulting foam properties and viscosity values of the investigated sample dispersions are published in a previous study [24].

\subsection{Foam Drying and Product Characterization}

Foams were dried by means of two different techniques: conventional vacuum drying (CVD) and microwave-assisted vacuum drying (MWVD).

For CVD, a pilot freeze dryer (Delta 1-24LSC; Martin Christ Gefriertrocknungsanlagen $\mathrm{GmbH}$, Osterode am Harz, Germany) was operated at a chamber pressure of $15 \mathrm{mbar}$ and a shelf temperature of $20^{\circ} \mathrm{C}$. The foams were dried for $16 \mathrm{~h}$. In case of foam collapse, drying was stopped $10 \mathrm{~min}$ after the collapse occurred.

For MWVD, the microwave drying plant $\mu$ Vac0150fd (Püschner GmbH \& Co. KG, Schwanewede, Germany) was used. Process control was conducted with the software 
$\mu$ WaveCAT (Püschner GmbH \& Co. KG, Schwanewede, Germany). A typical MWVD process is shown in Figure 1. Similar to CVD, MWVD was performed at 15 mbar. The maximum temperature was limited to $20^{\circ} \mathrm{C}$, and a microwave power input of $80 \mathrm{~W}$ was set. The drying process was stopped $10 \mathrm{~min}$ after foam collapse or when no mass loss was detected during 10 consecutive minutes. For successfully dried samples, the drying time was between 45 and $90 \mathrm{~min}$. The appearance of the resulting product structures was optically described.

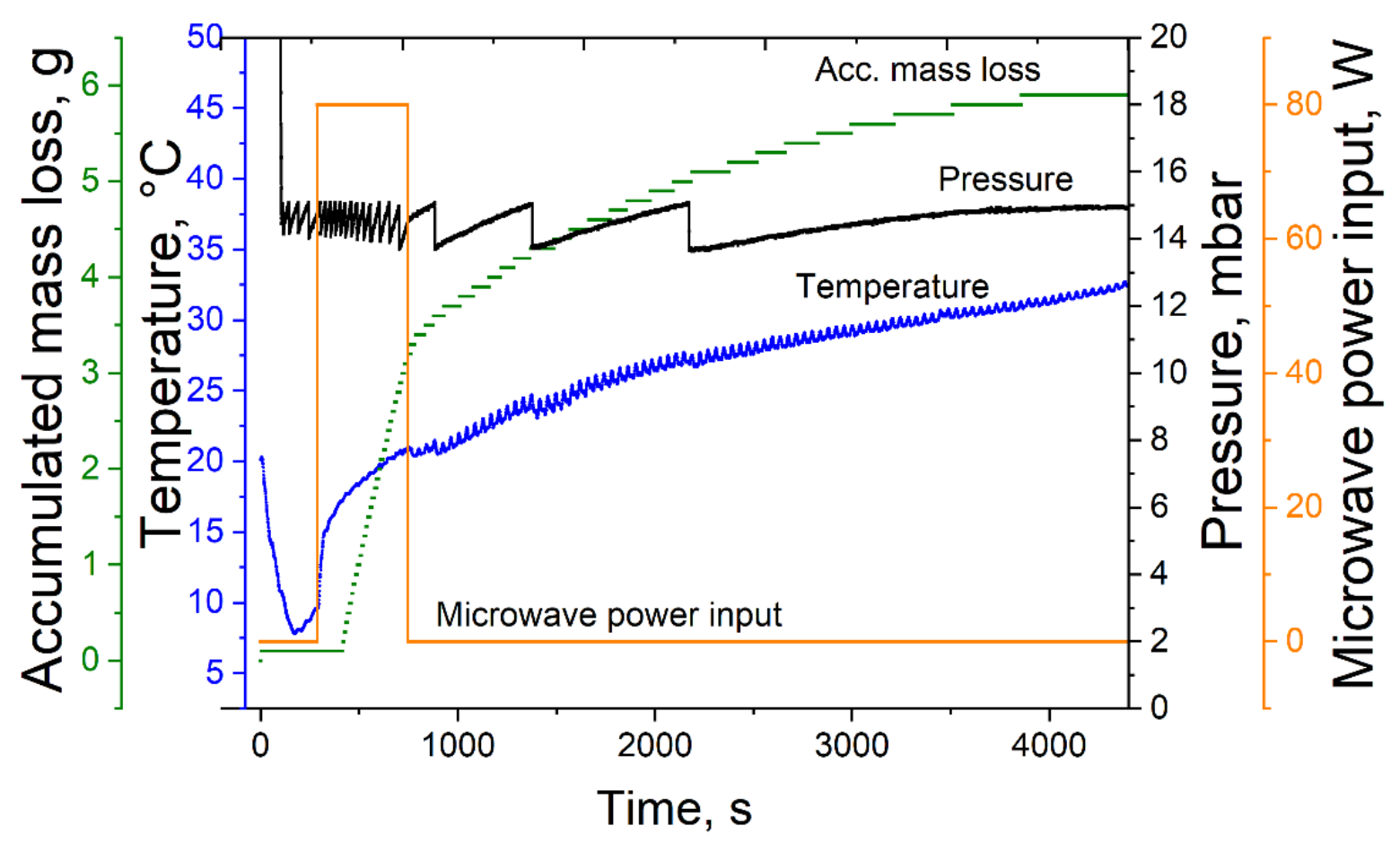

Figure 1. Exemplary microwave-assisted foam vacuum drying process at 15 mbar, $80 \mathrm{~W}$ microwave power input and a sample temperature of $20^{\circ} \mathrm{C}$.

\section{Results and Discussion}

\subsection{Foam Drying}

For the investigation of the interrelation between foam structure and drying behavior as a function of the heating technique, foams were prepared from polysorbate 80 at $3.0 \% w / w$ in combination with $10-40 \% w / w$ maltodextrin (MD). Thereby, an increase in the MD concentration led to an increase in the sample dispersion viscosity, which in turn resulted in a decrease in the overrun as well as the bubble size of the formed foams (results not shown). In terms of heating method, the foams were either subjected to conventional vacuum drying (CVD) or microwave-assisted vacuum drying (MWVD). For both drying processes, a maximum product temperature of $20^{\circ} \mathrm{C}$ was set. Overall, this approach gave the opportunity to distinguish heating method-specific effects and to identify relevant product properties for a successful drying process.

Figure 2 shows the different product structures obtained after either CVD or MWVD as a function of the MD concentration. Overall, both drying techniques, as well as MD content, had a clear impact on the appearance of the resulting product structures. For both drying techniques, foam structure preservation increased with increasing MD concentration, though CVD was clearly superior to MWVD. In the latter case, all samples collapsed during the drying process, yielding highly viscous liquids at the bottom of the sample containers. By contrast, by CVD, a foam-like structure was observed for samples containing 30 or $40 \% \mathrm{MD}$, whereas for 10 and $20 \% \mathrm{MD}$, foam collapse occurred during the first $30 \mathrm{~min}$ of drying. A better foam preservation during vacuum drying as a result of saccharide 
addition was also observed by Jangle and Pisal [25] investigating the impact of sucrose and mannitol on the vacuum foam drying of bovine serum albumin. The necessary amount of saccharide to obtain a foamy structure after the drying was comparable $(>20 \% w / v)$. This is interesting because the viscosity of a $20 \%$ sucrose dispersion is expected to be lower than a $20 \%$ maltodextrin dispersion, and the viscosity should preserve the foam structure [9]. A reason for this difference might be that in the recent study, foam formation was conducted before the drying process was started. Thereby, the foam was destabilized while the pressure was decreased, whereas, in the study of Jangle and Pisal, the vacuum step was necessary to form the foam. Hence, in our study, the foam expanded even before it got heated, which resulted in higher mechanical stress.
MD (\%) 10 20 30 40

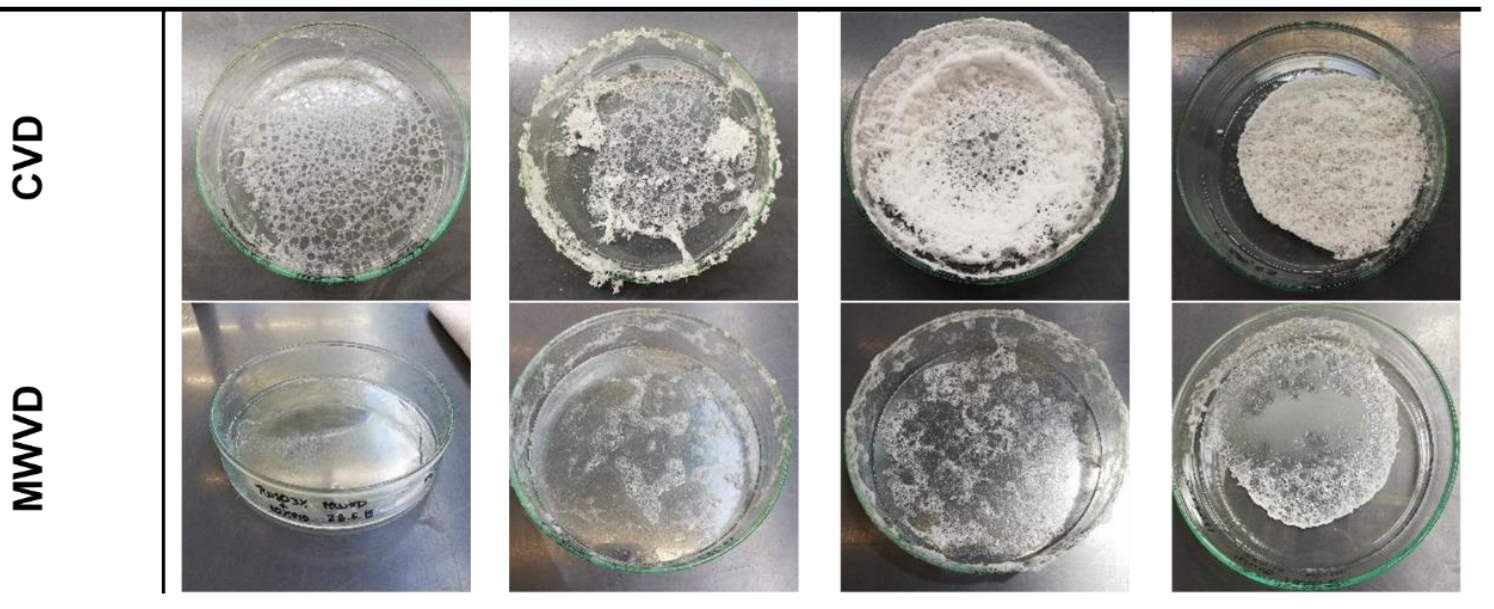

Figure 2. Impact of maltodextrin (MD) concentration and drying method on the appearance of the resulting product structures.

Overall, the results show that CVD was better suited to preserve foam structures from polysorbate 80 in combination with MD than MWVD. This raised the question of the specific relationship between the type of energy input and the surface properties of polysorbate 80 with MD. In this context, this study aimed at distinguishing properties being relevant for structure preservation and successful drying. In order to close this knowledge gap, surface tension and surface dilatational properties, as well as dielectric properties of the sample dispersions, were determined.

\subsection{Surface Tension and Surface Dilatational Properties of the Bulk Phase}

Firstly, the evolution of surface tension of the different sample dispersions (i.e., polysorbate 80 with $0-40 \% \mathrm{MD}$ ) was measured for a period of $7200 \mathrm{~s}$. The obtained results are displayed in Figure 3. The surface activity refers to the rate of initial surface tension decrease [26], which was calculated from the slope of the surface tension within the first $5 \mathrm{~s}$. In general, all sample dispersions exhibited a relatively high surface activity, which is characterized by the fact that quasi-equilibrium surface tension was almost reached within the first few seconds. The slight further decrease in surface tension over time was considered rather insignificant as compared to the very initial decrease. 


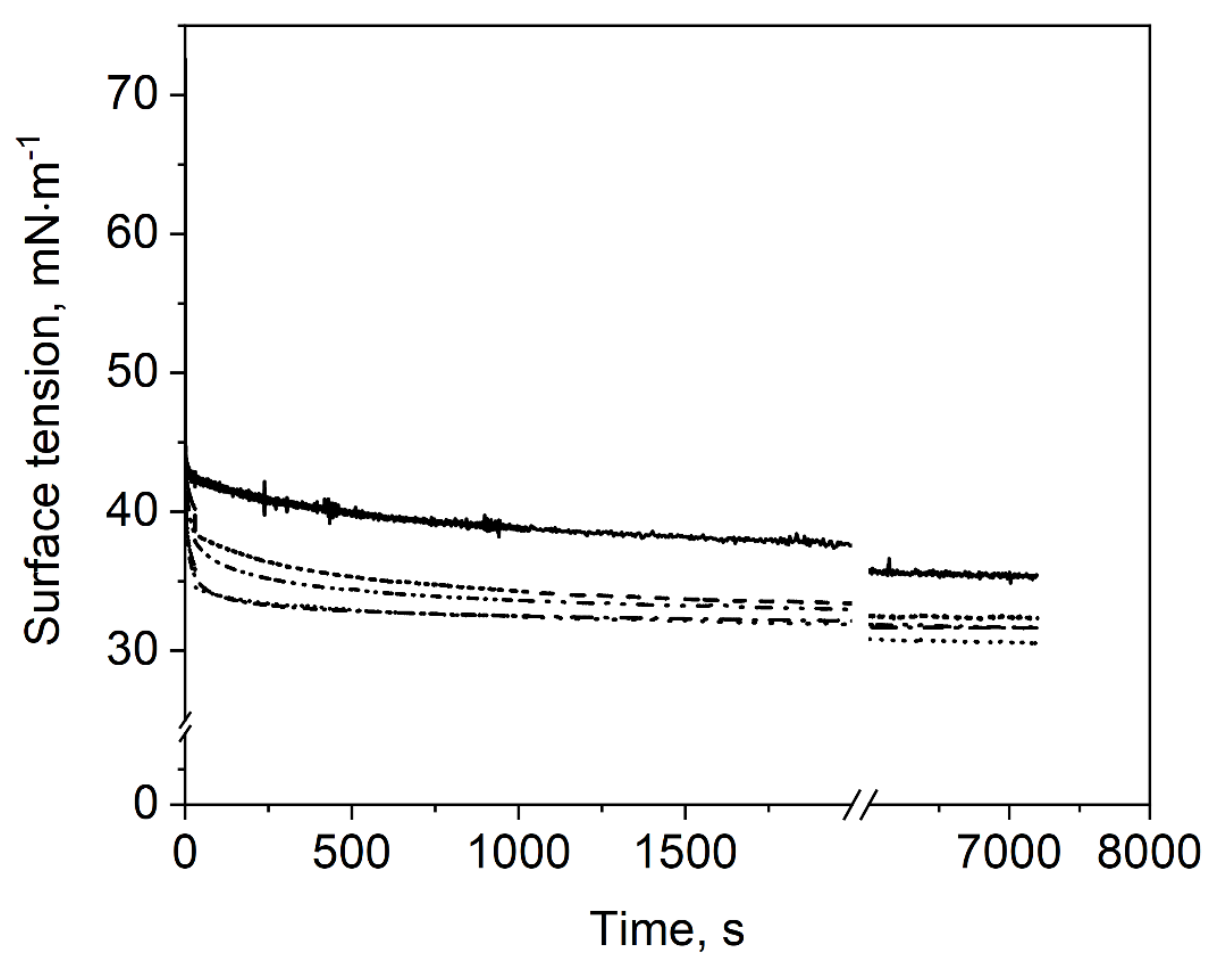

Figure 3. Impact of maltodextrin concentration on the time-dependent surface tension of polysorbate 80: (-) 0\% MD, (- -) 10\% MD, (...) 20\% MD, (--) 30\% MD, (-.-) 40\% MD.

It was observed that samples with MD showed higher surface activity and lower surface tension than the reference sample dispersion without MD. The surface activity increased from -6.0 at $0 \% \mathrm{MD}$ to $-7.4 \mathrm{mN} \cdot \mathrm{m}^{-1} \cdot \mathrm{s}^{-1}$ with $30 \% \mathrm{MD}$. The $40 \% \mathrm{MD}$ samples showed a slightly lower value of $-6.8 \mathrm{mN} \cdot \mathrm{m}^{-1} \cdot \mathrm{s}^{-1}$. Quasi-equilibrium surface tension of samples containing MD was around $33 \mathrm{mN} \cdot \mathrm{m}^{-1}$, whereas for $0 \% \mathrm{MD}$, a value of $37 \mathrm{mN} \cdot \mathrm{m}^{-1}$ was obtained. A possible explanation for this behavior could stem from the presence of surface-active molecules such as native starch lipids and proteins, originating from the maltodextrin manufacturing process $[27,28]$. However, the surface tension of MD dispersions with an MD content between 10 and $40 \%$ and without polysorbate 80 was reported to be in equilibrium between 71.2 and $72.5 \mathrm{mN} \cdot \mathrm{m}^{-1}$ [24]. Hence, the contamination of MD with surface-active components seems not to be the reason for the observed differences in surface tension. Therefore, we assume that the high viscosity of $40 \% \mathrm{MD}$ samples inhibited the polysorbate to move to the new surface and was therefore the reason for the slightly lower surface activity. Further, strong hydrogen bonds and hydrophobic interactions between polysorbate 80 and MD may change the surface activity of polysorbate 80 as also suggested by Semenova et al. [29]. Thereby, the surfactant might be modified via non-covalent interaction by extending the ethylene oxide group of polysorbate. Consequently, polysorbate $80-\mathrm{MD}$ mixtures could lower the surface tension more than samples which contain only polysorbate 80 . On the other hand, this might also be the reason for the slower adsorption of samples with $40 \% \mathrm{MD}$, as the interactions might result in a steric repulsion at the surface in the presence of high carbohydrate concentrations.

The lowest surface tension was obtained for sample dispersions containing 10 or $20 \% \mathrm{MD}$, whereas the surface tension was slightly higher for sample dispersions with 30 and $40 \% \mathrm{MD}$. This could be due to the increasing viscosity of the investigated sample dispersions with increasing MD concentration. Thereby, diffusion and adsorption of the surface-active molecules at the air/water interface might be hindered, resulting in a higher surface tension within the time frame investigated. Nevertheless, MD had an enhancing effect on surface activity and surface tension as compared to the reference sample dispersion without MD. 
Overall, the results show that surface activity and surface tension are not indicative for structure preservation during drying (Figure 2).

Besides surface tension, the different sample dispersions were also characterized in terms of their surface dilatational properties. Surface dilatational properties are deemed indicative of surface film stability, which was assumed to be of importance in view of gas expansion under vacuum as well as bubble deformation during drying. The results on surface dilatational elasticity $E^{\prime}$ and surface dilatational viscosity $E^{\prime \prime}$ are shown in Figure 4 as a function of the MD concentration. In terms of $E^{\prime \prime}$, no clear dependence on the MD concentration could be observed, though values slightly increased with increasing MD concentration from about 3.5 to $5.8 \mathrm{mN} \cdot \mathrm{m}^{-1}$. Nonetheless, the very slight increase in $E^{\prime \prime}$ could be due to the increased total solids content of the sample dispersions with increasing MD concentration, which in turn showed a positive effect during drying. Unlike surface tension, surface dilatational properties and especially $E^{\prime}$ strongly depended on the MD content. By comparison, results on $E^{\prime}$ followed a " $u$ " shape with a minimum at $25 \% \mathrm{MD}$. Thereby, the relative change in surface dilatational elasticity as a function of the MD concentration was quite high, with absolute values starting at $20.1 \mathrm{mN} \cdot \mathrm{m}^{-1}$ at $0 \%$ $\mathrm{MD}$, decreasing to $7.5 \mathrm{mN} \cdot \mathrm{m}^{-1}$ at $25 \% \mathrm{MD}$ and increasing again towards $21.7 \mathrm{mN} \cdot \mathrm{m}^{-1}$ at $40 \% \mathrm{MD}$. This can also be described by $\tan (\varphi)$, where 0 represents a perfect elastic behavior of the film (Table 1) [20,30]. The surface showed most elastic behavior at low $(\tan (\varphi)=0.2-0.24$ at $0-5 \% \mathrm{MD})$ and high $\mathrm{MD}(\tan (\varphi)=0.28$ at $40 \% \mathrm{MD})$, while for $20 \% \mathrm{MD}$, the surface exhibited the lowest elasticity $(\tan (\varphi)=0.53)$. However, in comparison with protein-stabilized systems, the obtained $\tan (\varphi)$ for our system is quite high. For example, Beaza et al. [30] investigated the influence of different additives to $\beta$-lactoglobulin and obtained $\tan (\varphi)$ values of $<0.2$. An explanation for this difference might be protein network formation by means of intermolecular interactions between protein molecules such as electrostatic interactions or hydrogen bonds. By contrast, small non-ionic surfactants such as polysorbate 80 are mainly interacting via hydrogen bonds.

Overall, the surface of polysorbate-stabilized foams showed low elastic behavior, which could be problematic regarding the high mechanical stress during the drying process.

However, no clear correlation could be resolved between drying performance in terms of structure preservation and surface dilatational elasticity.

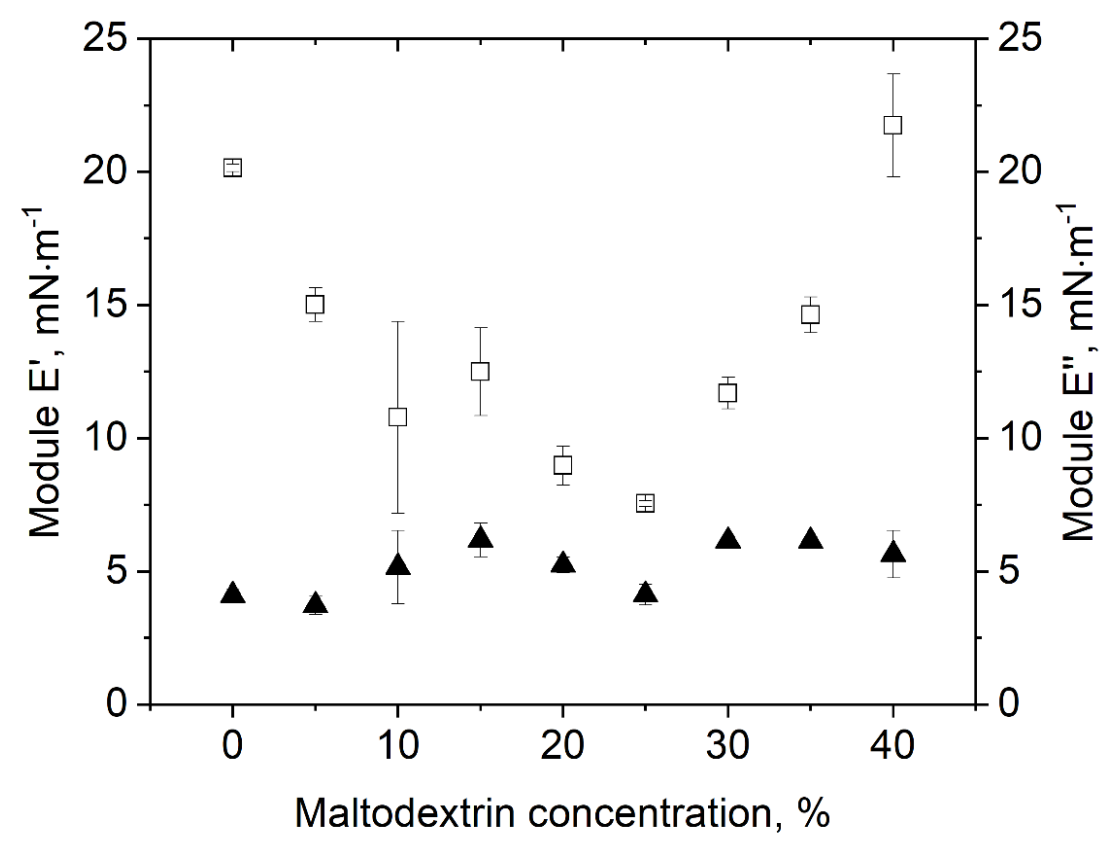

Figure 4. Impact of MD concentration on surface dilatational properties of polysorbate 80: ( $\square$ ) surface dilatational elasticity $E^{\prime} ;(\boldsymbol{\Lambda})$ surface dilatational viscosity $E^{\prime \prime}$ at an amplitude of $500 \%$. 
Table 1. Calculated $\tan (\varphi)$ of samples with $3 \%$ polysorbate 80 and an MD content between 0 and $40 \%$ at $20{ }^{\circ} \mathrm{C}$ and an amplitude of $500 \%$.

\begin{tabular}{cccccccccc}
\hline $\mathbf{c ( M D )}, \mathbf{\%}$ & $\mathbf{0}$ & $\mathbf{5}$ & $\mathbf{1 0}$ & $\mathbf{1 5}$ & $\mathbf{2 0}$ & $\mathbf{2 5}$ & $\mathbf{3 0}$ & $\mathbf{3 5}$ & $\mathbf{4 0}$ \\
\hline $\tan (\varphi),-$ & 0.20 & 0.24 & 0.45 & 0.46 & 0.53 & 0.50 & 0.48 & 0.40 & 0.28 \\
\hline
\end{tabular}

The effect of better stability at higher total solid content could be explained as such that the high viscosity of the bulk phase prevents the bubbles to expand too quickly. During drying, the lamellae become very thin, and the surface area increases. This in turn might result in the occurrence of holes in the surface films whereby foam collapse is triggered, eventually. The higher MD content could slow this down and enable the foaming agent to cover the surface quickly enough to prevent foam collapse. In terms of conventional vacuum drying, an MD concentration of $\geq 30 \%$ seemed favorable in terms of formation of elastic surface films, resulting in structure preservation during drying.

However, the results do not explain the observed differences between CVD and MWVD processes. Therefore, it was considered necessary to investigate in more depth the microwave-specific properties of the different sample dispersions. Therewith, it was aimed to better understand the underlying mechanisms allowing for specific conclusions regarding future product design.

\subsection{Dielectric Properties of the Bulk Phase}

The dielectric properties of polysorbate 80 dispersions with various contents of MD are shown in Figure 5. The dielectric constant decreased with increasing sugar concentration, while the dielectric loss factor slightly increased. A comparable trend for increasing carbohydrate concentration was also observed by Roebuck et al. [31] during their investigation of dielectric properties of carbohydrates using a microwave frequency of $3 \mathrm{GHz}$. They stated that the reason for the decrease in the dielectric constant was due to fewer polarizable dipole moments due to less free water in the samples. The increase in the dielectric loss factor with increasing carbohydrate content can be explained by a better stabilization of hydrogen bonds in the presence of carbohydrates. Haggis et al. [32] stated that due to better stabilized hydrogen bonds in the presence of organic molecules, the relaxation frequency of water is lowered, whereby the dielectric loss increases. Therefore, our findings are in accordance with our expectations and findings from the literature.

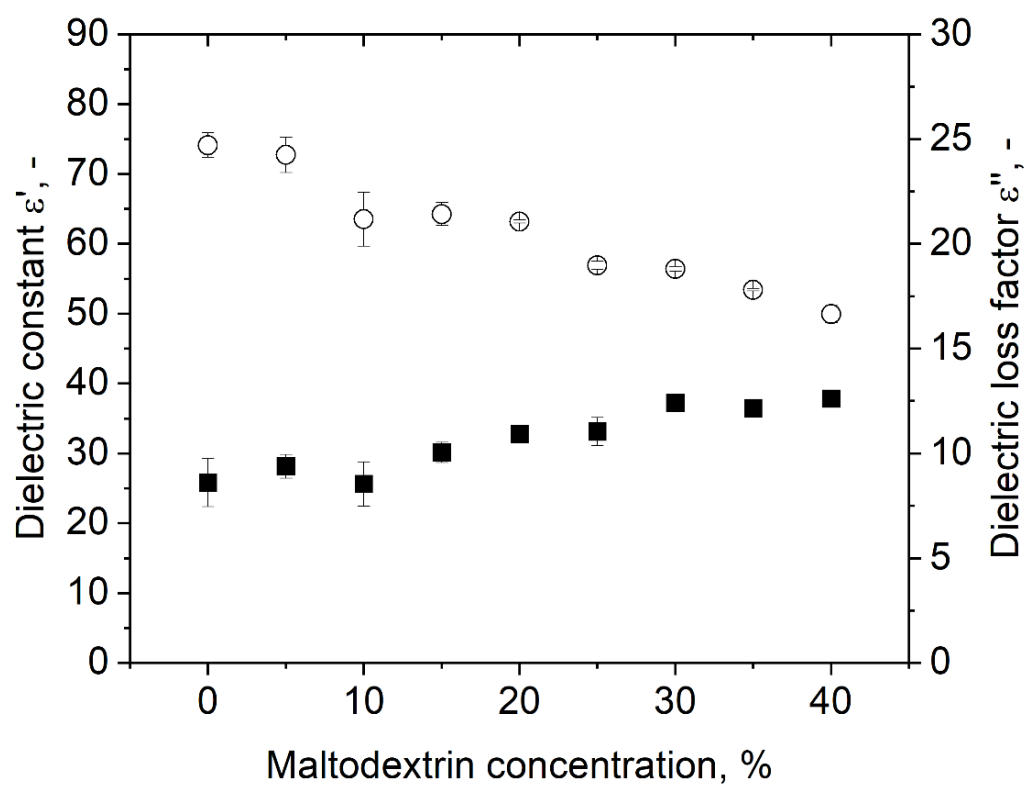

Figure 5. Impact of MD concentration on the dielectric properties of dispersions with $3 \%$ polysorbate 80. Symbols: $(\bigcirc)$ dielectric constant $\varepsilon^{\prime},(\mathbf{\square})$ dielectric loss $\varepsilon^{\prime \prime}$. 
As a result of the increasing dielectric loss and decreasing dielectric constant, the value for $\tan (\delta)$, which is a parameter for the ability of the material to converse radiation power into heat, increased (Table 2). Therefore, the samples were heated more efficiently with the higher sugar content. However, this might be problematic for the uniformity of the heating process: the more efficient the heating, the lower the penetration depth according to Equation (5). For the investigated samples, the penetration depth of the bulk decreased from 7.67 at $0 \% \mathrm{MD}$ to $3.84 \mathrm{~cm}$ at $40 \% \mathrm{MD}$. Therefore, the characteristics of the heating are less volumetric with increasing MD because more energy is converted into heat within a shorter distance. As a result, the probability of hot and cold spot formation is increased. The bubbles are assumed to not be heated extensively because the bulk phase converts the energy much more efficiently than the air inside the bubbles. Consequently, the penetration depth into the foam will increase with higher overrun values, which means that the differences in the heating pattern are expected to be even higher. However, no differences in hot spot formation were detected, which was contributed to the foam expansion during the drying process.

Table 2. Calculated $\tan (\delta)$ for dispersions with $3 \%$ polysorbate 80 and an MD content between 0 and $40 \%$ at $20{ }^{\circ} \mathrm{C}$.

\begin{tabular}{cccccccccc}
\hline $\mathrm{c}(\mathrm{MD}), \boldsymbol{\%}$ & $\mathbf{0}$ & $\mathbf{5}$ & $\mathbf{1 0}$ & $\mathbf{1 5}$ & $\mathbf{2 0}$ & $\mathbf{2 5}$ & $\mathbf{3 0}$ & $\mathbf{3 5}$ & $\mathbf{4 0}$ \\
\hline $\tan (\delta),-$ & 0.12 & 0.13 & 0.14 & 0.16 & 0.17 & 0.20 & 0.22 & 0.23 & 0.26 \\
\hline
\end{tabular}

In Figure 6, the resonant frequency of polysorbate 80 dispersions with an MD content between 0 and $40 \%$ is shown. It was observed that with increasing sugar content, the resonant frequency shifted from $1951.3 \pm 5.7$ to $2039.2 \pm 5.6 \mathrm{MHz}$. This seems to be plausible, as the addition of organic molecules increases the resonant frequency of water [33].

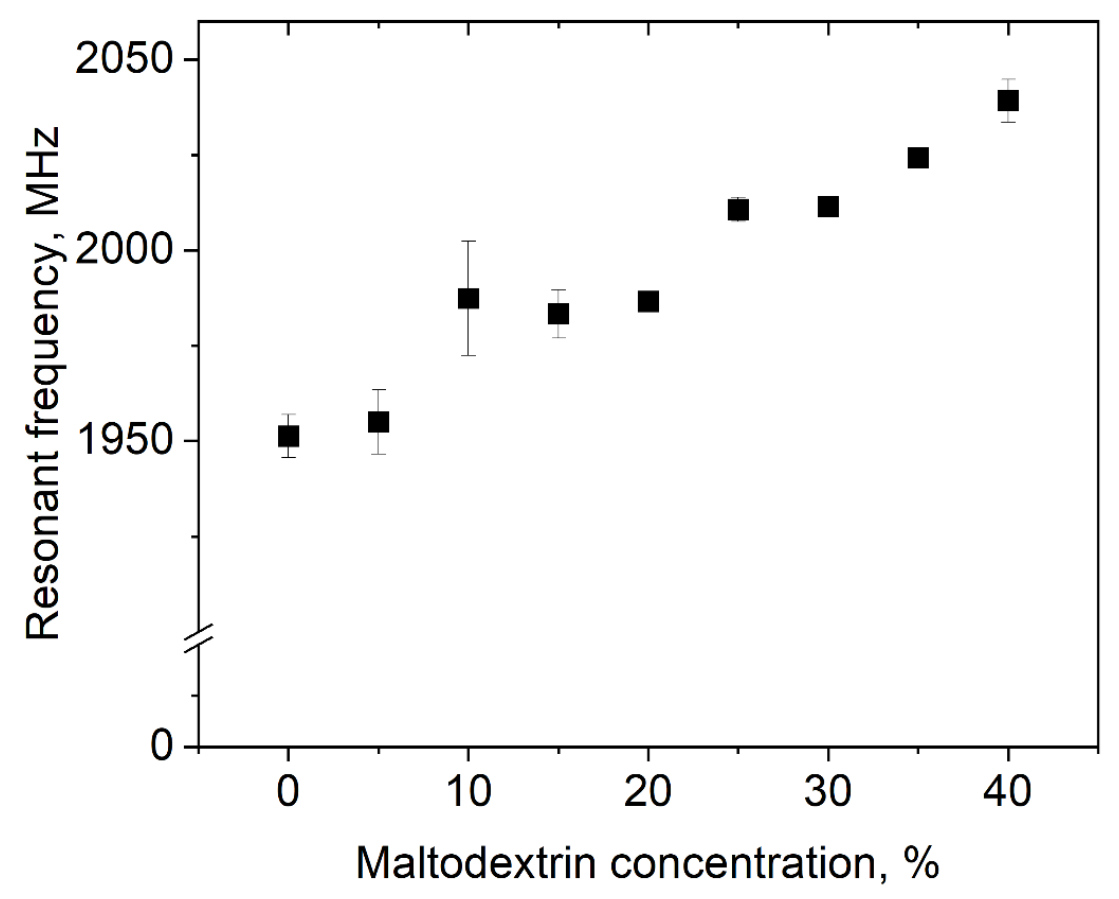

Figure 6. Impact of MD concentration on the resonant frequency of dispersions with $3 \%$ polysorbate 80.

Summing up the results on the dielectric properties of the dispersion, the samples were able to convert radiation more efficiently into heat at higher sugar contents. The faster and concentrated heating resulted in harsher heating conditions. Nevertheless, we observed better preservation of shape and structure at higher MD contents. Hence, the 
dielectric properties of the complex dispersion do not explain the collapse of the foam during the MWVD process.

In order to more deeply address this question, different concentrations of MD without a foaming agent (i.e., polysorbate 80 ) were tested (data not shown), but they did not give a hint for the drying behavior of the samples. Hence, a dispersion of $100 \%$ foaming agent was examined in view of its dielectric properties. In Figure 7, the complex reflection coefficient $S_{11}$ depending on the frequency is shown. This value represents the interactions of a material with the electromagnetic wave if it is impinged with a certain frequency. The frequency where the interactions are the strongest is called resonant frequency, which is at $2445 \mathrm{MHz}$ for the pure surfactant. This matches the frequency band which the microwave drying plant emits to apply power to the sample (i.e., $2450 \mathrm{MHz} \pm 50 \mathrm{MHz}$ ). Therefore, it appears that a so-called frequency catastrophe happened at the air/water interface, as schematically shown in Figure 8. Due to the overlapping microwave and resonant frequencies, the ethylene group of the surfactant is polarized very efficiently and at a high frequency. Further, interactions between water and the ethylene groups of polysorbate might be affected by the radiation [34], which might result in lower interface stability. Due to the high applied power, the movement of the surfactant caused hole formation within the surfactant layer. This in turn resulted in a collapse of the foam structure during microwave application.

For high sugar concentrations and, consequently, thick lamellae, this effect can be suppressed by the high viscosity of the bulk phase. However, due to the quick expansion of the air bubbles during the vacuum and heating process, it could be that the counteracting forces become too weak. In a first step, the air bubbles would show coalescence with other bubbles until the necessary amount of surfactant to stabilize the interface is too low. Then, the bubble and the foam would collapse.

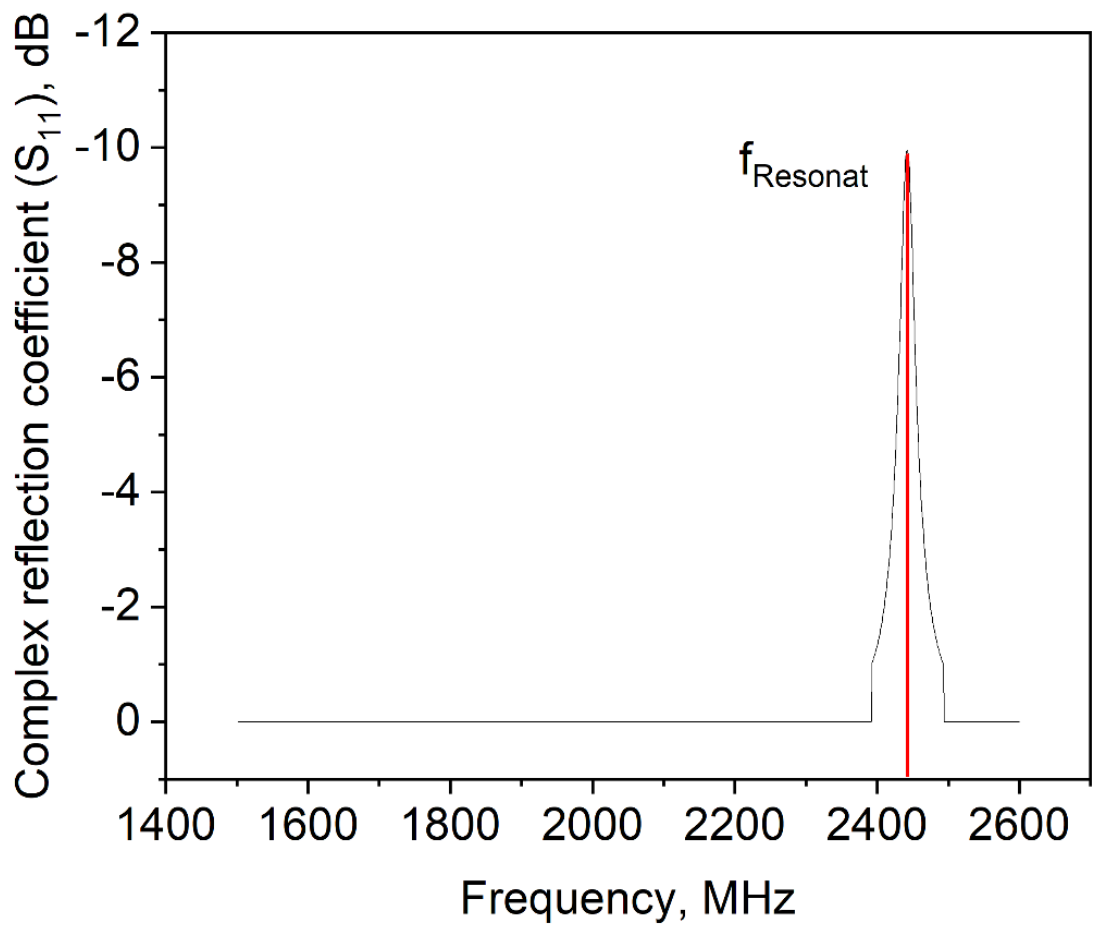

Figure 7. Complex reflection coefficient $S_{11}$ of pure polysorbate 80 . 


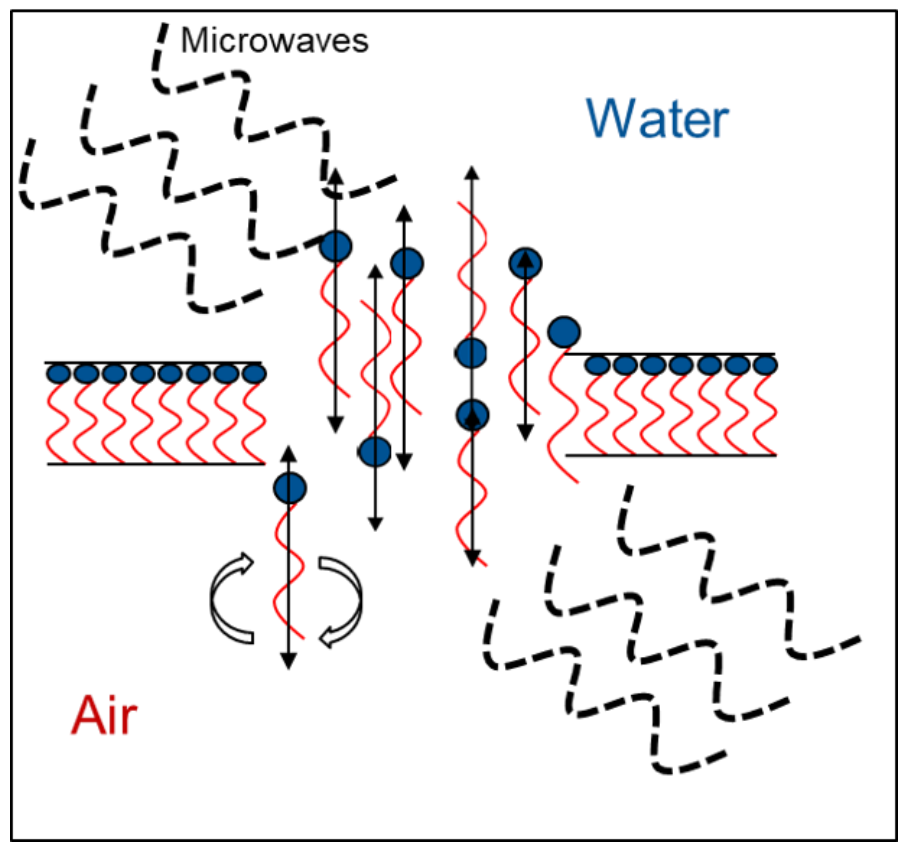

Figure 8. Schematic representation of the behavior of polysorbate 80 at the air/water interface during foam drying.

Observations during the drying process showed that the foam started to collapse when the microwave power was on and stopped decaying right after turning the microwave power off (Figure 1). This indicates strongly that the resonant frequency of the foaming agent had a strong impact on the foam decay and not the overall dielectric properties of the bulk phase. Finally, the dielectric properties of polysorbate 80 seem to be one of the main reasons for the foam decay during MWVD.

\section{Conclusions}

In this study, the behavior of polysorbate 80-maltodextrin foams during vacuum drying processes with different heating methods was investigated. It was shown that there is not one single parameter that is overall important to achieve a successful drying process. Nevertheless, surface dilatational rheology seems an important parameter because a certain elasticity $(\tan (\varphi)<0.4)$ of the surface is mandatory to withstand the mechanic stress during the evacuation of the drying plant. For the case of MWVD, it was not clear if just the dielectric properties of the surfactant had this massive impact on foam stability during the drying process. However, the results indicate that the dielectric properties of the foaming agent and the surface dilatational rheology of the complex system are responsible for its behavior during microwave-assisted processes, which is a new insight related to microwave-assisted drying. Further, a certain ratio of $E^{\prime \prime} / E^{\prime}$ expressed as $\tan (\varphi)<0.2$ was shown to be necessary for successful drying with MWVD, and the resonant frequency of the foaming agent should not match the working frequency of the microwave drying plant $(2450 \mathrm{MHz} \pm 50 \mathrm{MHz})$. As the dielectric properties change with the temperature, it would be interesting to investigate these parameters at different, commonly used temperatures for vacuum drying. Further, it would be of interest to determine the importance of effects at the molecular level to better understand the behavior of foam during the drying process. Besides surfactants, proteins are commonly used as foaming agents, which is why we investigated the behavior of protein samples during MWVD in a second study. Further, it would be of interest to use other microwave sources to determine the specific effect of the microwave frequency. 
Author Contributions: P.K.: conceptualization, methodology, investigation, writing—original draft, writing—review and editing, visualization; U.K.: writing—review and editing, resources, supervision, funding acquisition; J.D.: conceptualization, writing —original draft, writing-review and editing. All authors have read and agreed to the published version of the manuscript.

Funding: This research project was supported by the German Ministry of Economics and Energy (via AiF) and the FEI (Forschungskreis der Ernährungsindustrie e.V., Bonn), Project AiF 18819 N. This work was supported by the Technical University of Munich (TUM) in the framework of Open Access Publishing Program.

Data Availability Statement: Data will be available upon request from the corresponding author.

Acknowledgments: The authors would like to thank Krüss GmbH for providing the DSA100R. The authors would like to thank Luísa Leitão for helping with the experimental data collection.

Conflicts of Interest: The authors declare no conflict of interest. The funders had no role in the design of the study; in the collection, analyses, or interpretation of data; in the writing of the manuscript, or in the decision to publish the results.

\section{References}

1. Ambros, S.; Bauer, S.A.W.; Shylkina, L.; Foerst, P.; Kulozik, U. Microwave-Vacuum Drying of Lactic Acid Bacteria: Influence of Process Parameters on Survival and Acidification Activity. Food Bioprocess Technol. 2016, 9, 1901-1911. [CrossRef]

2. Bauer, S.A.W.; Schneider, S.; Behr, J.; Kulozik, U.; Foerst, P. Combined influence of fermentation and drying conditions on survival and metabolic activity of starter and probiotic cultures after low-temperature vacuum drying. J. Biotechnol. 2012, 159, 351-357. [CrossRef]

3. de Jesus, S.S.; Filho, R.M. Optimizing Drying Conditions for the Microwave Vacuum Drying of Enzymes. Dry Technol. 2011, 29, 1828-1835. [CrossRef]

4. McLoughlin, C.M.; McMinn, W.A.M.; Magee, T.R.A. Microwave-Vacuum Drying of Pharmaceutical Powders. Dry. Technol. 2003, 21, 1719-1733. [CrossRef]

5. Gehrmann, D.; Esper, G.J.; Schuchmann, H. Trocknungstechnik in der Lebensmittelindustrie, 1st ed.; Behr: Hamburg, Germany, 2009.

6. Santivarangkna, C.; Wenning, M.; Foerst, P.; Kulozik, U. Damage of cell envelope of Lactobacillus helveticus during vacuum drying. J. Appl. Microbiol. 2007, 102, 748-756. [CrossRef]

7. Foerst, P.; Kulozik, U.; Schmitt, M.; Bauer, S.; Santivarangkna, C. Storage stability of vacuum-dried probiotic bacterium Lactobacillus paracasei F19. Food Bioprod. Process. 2012, 90, 295-300. [CrossRef]

8. Ambros, S.; Dombrowski, J.; Boettger, D.; Kulozik, U. The Concept of Microwave Foam Drying Under Vacuum: A Gentle Preservation Method for Sensitive Biological Material. J. Food Sci. 2019, 84, 1682-1691. [CrossRef]

9. Ambros, S.; Dombrowski, J.; Boettger, D.; Kulozik, U. Structure-Function-Process Relationship for Microwave Vacuum Drying of Lactic Acid Bacteria in Aerated Matrices. Food Bioprocess Technol. 2019, 12, 395-408. [CrossRef]

10. Rajkumar, P.; Kailappan, R.; Viswanathan, R.; Raghavan, G.S.V. Drying characteristics of foamed alphonso mango pulp in a continuous type foam mat dryer. J. Food Eng. 2007, 79, 1452-1459. [CrossRef]

11. Ratti, C.; Kudra, T. Drying of Foamed Biological Materials: Opportunities and Challenges. Dry. Technol. 2006, $24,1101-1108$. [CrossRef]

12. Sankat, C.K.; Castaigne, F. Foaming and drying behaviour of ripe bananas. LWT 2004, 37, 517-525. [CrossRef]

13. Patino, J.M.R.; Sánchez, C.C.; Niño, M.R.R. Implications of interfacial characteristics of food foaming agents in foam formulations. Adv. Colloid Interface Sci. 2008, 140, 95-113. [CrossRef]

14. Georgieva, D.; Cagna, A.; Langevin, D. Link between surface elasticity and foam stability. Soft Matter 2009, 5, $2063-2071$. [CrossRef]

15. Guo, W.; Zhu, X. Dielectric Properties of Red Pepper Powder Related to Radiofrequency and Microwave Drying. Food Bioprocess Technol. 2014, 7, 3591-3601. [CrossRef]

16. Jaya, S.; Das, H. Effect of maltodextrin, glycerol monostearate and tricalcium phosphate on vacuum dried mango powder properties. J. Food Eng. 2004, 63, 125-134. [CrossRef]

17. Jaya, S.; Das, H.; Mani, S. Optimization of Maltodextrin and Tricalcium Phosphate for Producing Vacuum Dried Mango Powder. Int. J. Food Prop. 2006, 9, 13-24. [CrossRef]

18. Kubbutat, P.; Kulozik, U.; Dombrowski, J. Foam structure preservation during microwave-assisted vacuum drying: Significance of interfacial and dielectric properties of the bulk phase of the bulk phase of foams from whey protein isolate/maltodextrin dispersions. J. Food Eng. 2021, 110691. [CrossRef]

19. Lucasson, J.; van den Tempel, M. Dynamic measurements of dilational properties of a liquid interface. Chem. Eng. Sci. 1972, 27, 1283-1291. [CrossRef]

20. Conde, J.M.; Patino, J.M.R. Rheological Properties of Hydrolysates of Proteins from Extracted Sunflower Flour Adsorbed at the Air-Water Interface. Ind. Eng. Chem. Res. 2005, 44, 7761-7769. [CrossRef] 
21. Pueschner GmbH \& Co. KG, Dielectric Measurement Kit $\mu$ WaveAnalyser: Short Technical Notes. 2008. Available online: https://www.pueschner.com/downloads/product-news/werb_mwmess_techDaten_en.pdf (accessed on 9 April 2020).

22. Bart, J.C.J. Additives in Polymers: Industrial Analysis and Applications; John Wiley \& Sons Ltd.: Chichester, UK, 2005.

23. Adrian, R.; Patryk, W. Influence of water on the dielectric properties, electrical conductivity and microwave absorption properties of amorphous yellow dextrin. Cellulose 2019, 26, 2987-2998.

24. Kubbutat, P.; Kulozik, U. Interactions of Sugar Alcohol, Di-Saccharides and Polysaccharides with Polysorbate 80 as Surfactant in the Stabilization of Foams. Colloids Surf. A Physicochem. Eng. Asp. 2021, 616, 126349. [CrossRef]

25. Jangle, R.D.; Pisal, S.S. Vacuum Foam Drying: An Alternative to Lyophilization for Biomolecule Preservation. Indian J. Pharm. Sci. 2012, 74, 91-100. [CrossRef] [PubMed]

26. Marinova, K.G.; Basheva, E.S.; Nenova, B.; Temelska, M.; Mirarefi, A.Y.; Campbell, B.; Ivanov, I.B. Physico-chemical factors controlling the foamability and foam stability of milk proteins: Sodium caseinate and whey protein concentrates. Food Hydrocoll. 2009, 23, 1864-1876. [CrossRef]

27. Shogren, R.; Biresaw, G. Surface properties of water soluble maltodextrin, starch acetates and starch acetates/alkenylsuccinates. Colloids Surf. A 2007, 298, 170-176. [CrossRef]

28. Pycia, K.; Juszczak, L.; Gałkowska, D.; Socha, R.; Jaworska, G. Maltodextrins from chemically modified starches. Production and characteristics. Starch Stärke 2017, 69, 1600199. [CrossRef]

29. Semenova, M.G.; Belyakova, L.E.; Antipova, A.S.; Polikarpov, Y.N.; Klouda, L.; Markovic, A.; Il'in, M.M. Effect of maltodextrins on the surface activity of small-molecule surfactants. Colloids Surf. B 2003, 31, 47-54. [CrossRef]

30. Baeza, R.; Pilosof, A.M.R.; Sanchez, C.C.; Patino, J.M.R. Adsorption and rheological properties of biopolymers at the air-water interface. AIChE J. 2006, 52, 2627-2638. [CrossRef]

31. Roebuck, B.D.; Goldblith, S.A. Dielectric Properties of Carbohydrate-Waser Mixtures at Microwave Frequencies. J. Food Sci. 1972, 37, 199-202. [CrossRef]

32. Haggis, G.H.; Hasted, J.B.; Buchanan, T.J. The Dielectric Properties of Water in Solutions. J. Chem. Phys. 1952, $20,1452-1465$. [CrossRef]

33. Zhang, X.; Ruan, C.; Haq, T.U.; Chen, K. High-Sensitivity Microwave Sensor for Liquid Characterization Using a Complementary Circular Spiral Resonator. Sensors 2019, 19, 787. [CrossRef]

34. Epstein, B.R.; Foster, K.R.; Mackay, R.A. Microwave dielectric properties of ionic and nonionic microemulsions. J. Colloid Interface Sci. 1983, 95, 218-227. [CrossRef] 